\title{
AutoT\&T v.2: An Efficient and Versatile Tool for Lead Structure Generation and Optimization
}

Yan Li, ${ }^{\mathrm{a}}$ Zhixiong Zhao, ${ }^{\mathrm{a}}$ Zhihai Liu, ${ }^{\mathrm{a}}$ Minyi Su, ${ }^{\mathrm{a}}$ and Renxiao Wang ${ }^{\mathrm{a}, \mathrm{b} *}$

${ }^{a}$ State Key Laboratory of Bioorganic and Natural Products Chemistry, Collaborative Innovation Center of Chemistry for Life Sciences, Shanghai Institute of Organic Chemistry, Chinese Academy of Sciences, Shanghai 200032, People's Republic of China

${ }^{b}$ State Key Laboratory of Quality Research in Chinese Medicine, Macau Institute for Applied Research in Medicine and Health, Macau University of Science and Technology, Macau, People's Republic of China

* To whom all correspondence should be addressed: wangrx@mail.sioc.ac.cn

\section{Table of Contents}

Part I. Descriptions of Several Key Algorithms in AutoT\&T2 ….............................................2

Part II. Performance of AutoT\&T2 versus Other Two Build-Up Methods ...................................7

Part III. Additional Results Given by AutoT\&T2 in the Three Test Cases.................................... 12

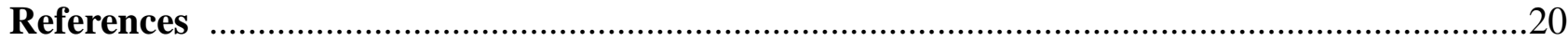




\section{Part I. Descriptions of Several Key Algorithms in AutoT\&T2}

Special note: The algorithms described here are common between AutoT\&T2 and the original AutoT\&T. For the sake of convenience, we will refer to them non-discriminatorily as "AutoT\&T" in the following sections.

\subsection{Algorithm for detecting matched bonds}

A basic step in the structural operation module of AutoT\&T is to detect the matched bonds between the lead molecule and a given reference molecule. Two algorithms are implemented in AutoT\&T for judging if two given bonds are geometrically overlapped, i.e. "matched". One is based on matching atom pairs; while the other is based on matching bond centers (see Table S1). Both of them require that the matched bonds should not locate on a ring. The atom-pair based algorithm considers the distances between both ends of the two given bonds ( $r_{1}$ and $\left.r_{2}\right)$; while the bond-center based algorithm considers the distance between the centers of two bonds $(d)$. The matching degree (MD) between two bonds is computed as:

$$
\begin{aligned}
& M D=\frac{r_{\text {cutoff }}-r_{1}}{r_{\text {cutoff }}} \times \frac{r_{\text {cutoff }}-r_{2}}{r_{\text {cutoff }}} \times \frac{\theta_{\text {cutoff }}-\theta}{\theta_{\text {cutoff }}} \\
& M D=\frac{d_{\text {cutoff }}-d}{d_{\text {cutoff }}} \times \frac{\theta_{\text {cutoff }}-\theta}{\theta_{\text {cutoff }}}
\end{aligned}
$$

Here, $r_{1}$ and $r_{2}$ are the distances between both ends of the two bonds; $r_{\text {cutoff }}$ denotes for the cutoff value of these distances; $d$ is the distance between the centers of two bonds, $d_{\text {cutoff }}$ denotes for the cutoff value for this distance; $\theta$ is the angle between the two bonds; $\theta_{\text {cutoff }}$ denotes for the cutoff value of this angle, which is the same for both algorithms. The possible values of matching degree range from 0 to 1 . A higher value indicates a better overlapping of the two bonds under examination. When using AutoT\&T, the distance and angle cutoff values can be set by the user.

If a pair of geometrically overlapped bonds is found between the lead molecule and a reference molecule, the fragments at both sides of this bond on the reference molecule may be used to replace the corresponding part on the lead molecule. However, if the fragment to be transplanted (on the reference) is identical to the one to be replaced (on the lead), obviously such an operation will not result in any new molecules. Thus, AutoT\&T considers such cases as invalid matched bonds and ignores them. The remaining matched pairs are ranked by their matching degrees in a decreasing 
order and are considered in structural operation.

Table S1. Summary of the two algorithms for detecting matched bonds

\begin{tabular}{|c|c|}
\hline Atom-pair based & Bond-center based \\
\hline $\begin{array}{c}r_{1} \\
\text { atom }\end{array}$ & $\begin{array}{l}\leftarrow \text { atom } \\
\text { atom }\end{array}$ \\
\hline $\begin{array}{l}\text { Matched conditions: } \\
\text { 1. Neither of the two bonds locates on a ring. } \\
\text { 2. Distances } \boldsymbol{r}_{1} \text { and } \boldsymbol{r}_{\mathbf{2}} \text { between each terminal } \\
\text { atom of two bonds are both smaller than } 1.0 \AA \text {. } \\
\text { 3. The angle } \boldsymbol{\theta} \text { between two bonds } \leq 15^{\circ} \text {. }\end{array}$ & $\begin{array}{l}\text { Matched conditions: } \\
\text { 1. Neither of the two bonds locates on a ring. } \\
\text { 2. Distance } \boldsymbol{d} \text { between each center of the two } \\
\text { bonds is smaller than } 0.5 \AA \text {. } \\
\text { 3. The angle } \boldsymbol{\theta} \text { between two bonds } \leq 15^{\circ} \text {. }\end{array}$ \\
\hline
\end{tabular}

\subsection{The PLP Scoring Function for Computing Binding Scores}

Computation of binding scores is needed in two ways in AutoT\&T. First, during fragment transplanting, only a fragment with a higher binding score on a reference molecule is allowed to replace a relevant fragment with a lower binding score on the lead molecule. Second, after each round of structural operation, the binding score of each newly generated ligand molecule is computed. Only those with a binding score higher than the lead molecule are kept for further consideration. Since AutoT\&T needs to process a large number of molecules in a single job, the desired scoring method is supposed to achieve a compromise between accuracy and speed. In fact, only a scoring function can fulfill this demand.

In AutoT\&T, the piecewise linear potential (PLP) scoring function [1] is used for computing binding scores. PLP is an empirical scoring function, which sums up distance-dependent potentials of the atom pairs between a protein and a ligand. It defines four types of heavy atoms on the protein and the ligand, i.e. hydrogen bond donor, hydrogen bond acceptor, hydrogen bond donor \& acceptor, and non-polar atom. The hydrogen bond interactions and steric interactions between these atoms are considered, which are calculated on the basis of the potential function shown in Figure S1. The interaction types and parameters are provided in Table S2 and Figure S1 respectively. 


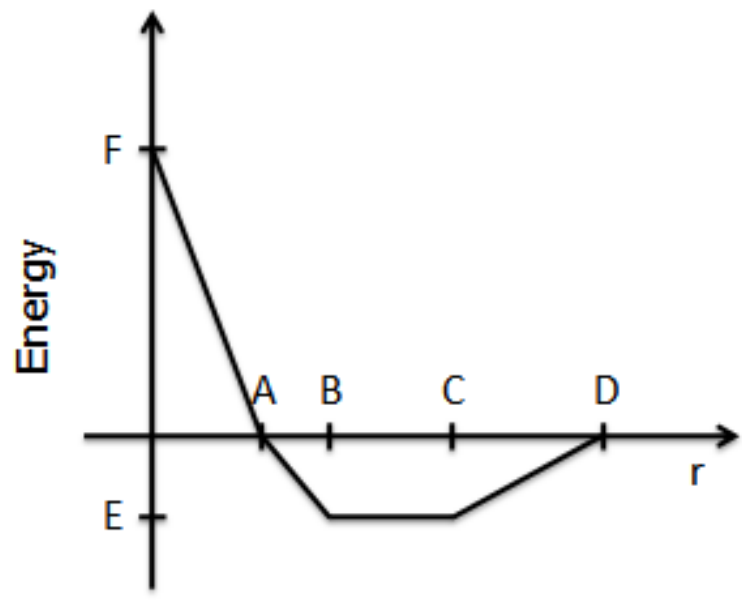

Figure S1. The stepwise potential function used in the PLP scoring function. Here A, B, C and D are distance cutoffs in $\AA$; $\mathrm{E}$ and $\mathrm{F}$ are binding scores in an arbitrary unit. For steric interactions, $\mathrm{A}=3.4, \mathrm{~B}=3.6$, $\mathrm{C}=4.5, \mathrm{D}=5.5, \mathrm{E}=-0.4, \mathrm{~F}=20.0$. For hydrogen bond interactions, $\mathrm{A}=2.3, \mathrm{~B}=2.6, \mathrm{C}=3.1, \mathrm{D}=3.4, \mathrm{E}=$ $-2.0, \mathrm{~F}=20.0$.

Table S2. The interaction types of different atom types on the protein and the ligand

\begin{tabular}{|c|c|c|c|c|c|}
\hline & & \multicolumn{4}{|c|}{ Atoms on the protein } \\
\hline & & $\begin{array}{l}\text { Hydrogen } \\
\text { bond donor }\end{array}$ & $\begin{array}{l}\text { Hydrogen } \\
\text { bond acceptor }\end{array}$ & $\begin{array}{l}\text { Hydrogen } \\
\text { bond donor \& } \\
\text { acceptor }\end{array}$ & $\begin{array}{l}\text { Non-polar } \\
\text { atom }\end{array}$ \\
\hline \multirow{4}{*}{$\begin{array}{c}\text { Atoms on the } \\
\text { ligand }\end{array}$} & $\begin{array}{l}\text { Hydrogen } \\
\text { bond donor }\end{array}$ & I & II & II & I \\
\hline & $\begin{array}{l}\text { Hydrogen } \\
\text { bond acceptor }\end{array}$ & II & $\mathrm{I}$ & II & I \\
\hline & $\begin{array}{l}\text { Hydrogen } \\
\text { bond donor \& } \\
\text { acceptor }\end{array}$ & II & II & II & I \\
\hline & $\begin{array}{l}\text { Non-polar } \\
\text { atom }\end{array}$ & $\mathrm{I}$ & $\mathrm{I}$ & $\mathrm{I}$ & $\mathrm{I}$ \\
\hline
\end{tabular}

I: steric interaction; II: hydrogen bond interaction

The PLP scoring function is chosen over other scoring functions due to its reasonable performance and technical advantages. First, some benchmarks of scoring functions [2,3] also suggest that PLP is among the top ones in terms of general performance. Second, PLP is a pure atom-based model. It is convenient to implement and also has a faster speed. Besides, it is possible with PLP to compute the binding score of any fragment in a molecule, which is important for AutoT\&T. 


\subsection{The RECAP Scheme for Assessing Synthetic Feasibility}

AutoT\&T constructs ligand molecules by assembling fragments. The resulting molecules are not always feasible for organic synthesis, which is actually a common problem to de novo design methods. The REtrosynthetic Combinatorial Analysis Procedure (RECAP) proposed by Lewell et al [4] is implemented in AutoT\&T to deal with this problem. According to this scheme, a total of 11 types of chemical bonds are defined to be "breakable”, each of which is related to a certain type of real chemical reaction (Figure S2). Breaking or recombining the chemical structures at these bonds may produce molecules that are more feasible for organic synthesis. RECAP analysis is optional in AutoT\&T. If enabled, whenever a pair of matched bonds is found between the lead molecule and a reference molecule, the program will check if this bond belongs to one of these 11 types defined in the RECAP scheme. If not, this matched bond pair will be ignored.

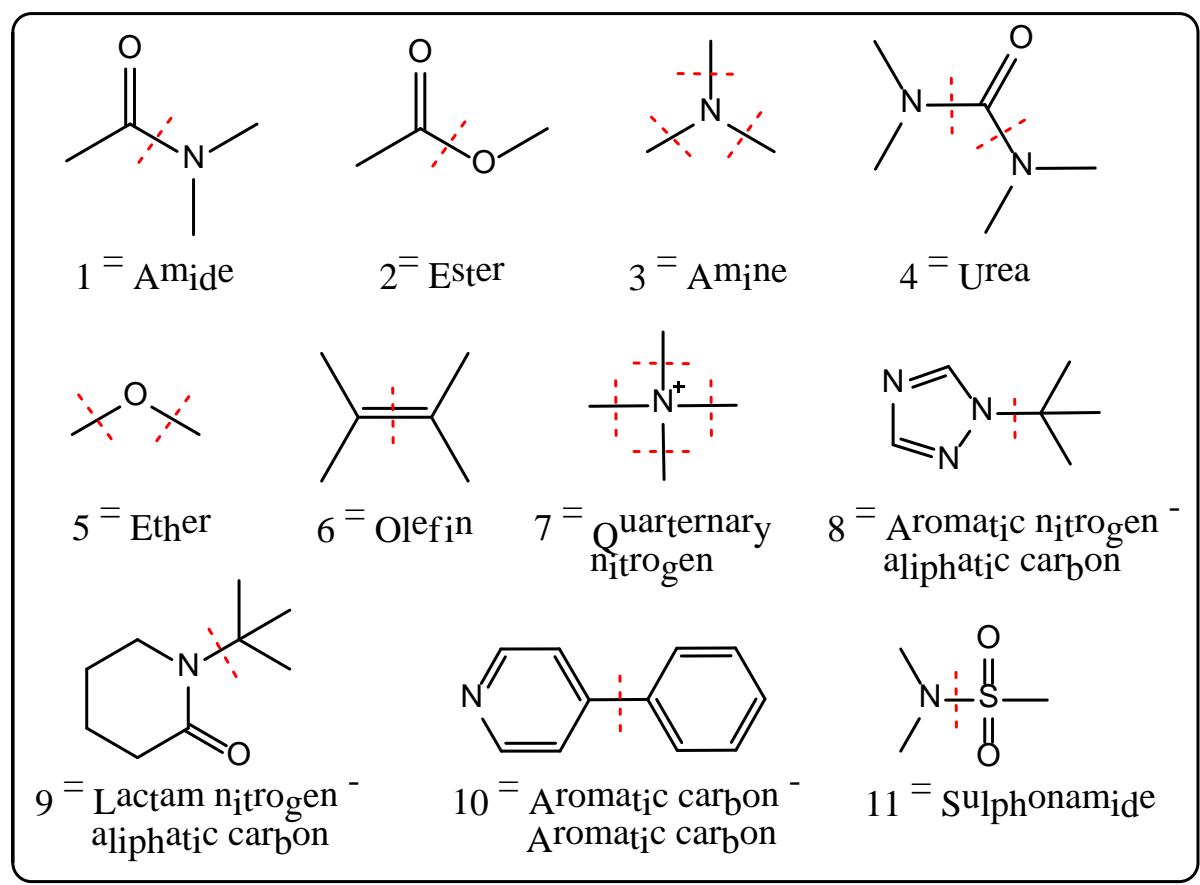

Figure S2. Eleven types of breakable chemical bonds defined in RECAP analysis

Introduction of the RECAP analysis takes the synthetic feasibility into account directly in structural operations. But it should be pointed out that the RECAP analysis is a very simple treatment. The user is encouraged to employ other more advanced methods for assessing the final outputs of AutoT\&T in terms of synthetic feasibility.

\subsection{The ECFP Fingerprints for Encoding Chemical Structures}

During the structural operations conducted by AutoT\&T, it is a frequent job to compare the structures of two molecules or fragments to judge if they are identical. This job can be done most 
efficiently by a 2D-fingerprint. The final outputs of AutoT\&T, typically thousands of molecules, are clustered by their structural similarity, which is also based on fingerprints. The extended connectivity fingerprints (ECFP) [5] is adopted in AutoT\&T for these purposes. Briefly, the initial fingerprint of each atom in a molecule is generated as a binary string via a hash function. It contains the information of seven invariants, including atomic number, type of this atom, number of heavy atoms attached to this atom, sum of chemical bonds formed with surrounding heavy atoms, formal charge, an indicator of chirality or stereo, and an indicator of isotope. After initialization, each heavy atom is taken in turn under consideration. The information of its attached heavy atoms is combined with the initial fingerprint of the atom at the center to form a new string. One can extend the coverage of the attached atoms and repeat the above operations. Each operation will update the fingerprints of each atom and the entire molecule. Generally, four iterations of such operation, i.e. ECFP_4, are enough for decreasing possible duplicated fingerprints effectively with reasonable computational efficiency. 


\section{Part II. Performance of AutoT\&T2 versus Other Two Build-Up Methods: A Test}

\section{Case}

Performance of AutoT\&T2 in the standard running mode, i.e. optimization of a single given lead molecule, was compared with other two conventional build-up methods implemented in commercial software. Here, one build-up method under test is the LUDI module (the "De Novo Evolution” module) in the Discovery Studio software (version 4.1, Accelrys Inc.), and the other is MUSE [6,7], which is supplied as a standalone program (version 4.0, Certara Inc.). It should be pointed out that although AutoT\&T2, LUDI, and MUSE are all de novo design methods, they use very different algorithms, support different running modes, and rely on different fragment libraries (or reference molecules in the case of AutoT\&T2). It is thus difficult to make a rigorous comparison of their performance. In a hope to make a meaningful comparison, we applied these three methods to conduct the same type of optimization job, i.e. "growing" fragments from a designated optimization site on a given lead molecule. Settings of the test case and the obtained results are described below.

In our previous study, a test case in which p38 MAP kinase was chosen as the molecular target was used to validate the original AutoT\&T [8]. Here, the same test case was employed to make a comparison between AutoT\&T2, LUDI and MUSE. The target structure was again retrieved from PDB entry 1W82, which is a complex formed between p38 MAP kinase and a small-molecule inhibitor [9]. The same lead molecule used in our previous study was used again in this case (Figure S3). This particular compound was selected because its size is relatively small and its potency is only at the micromolar range $\left(I C_{50}=40 \mu \mathrm{M}\right)$ so there is still enough room for further optimization. The binding pose of this lead molecule was generated by using the GOLD molecular docking software (version 5.2, Cambridge Crystallography Data Center). As in our previous study, the segment containing the indole moiety was kept as the core fragment; while the benzoic amide moiety was designated to be replaced in optimization (Figure S3).

To set up the job for AutoT\&T2, the C-N bond connecting the core fragment and the benzoic amide moiety was set as the optimization site, where fragment transplantation would be conducted. As the setting used in our previous study, a total of 1000 molecules randomly selected from the Available Chemical Directory database (ACD) were used as the reference molecules. Their binding poses inside the binding pocket on the target protein were also generated by the GOLD software. The PLP scoring function implemented in AutoT\&T2 was used for evaluating protein-ligand interactions. Note that since only one optimization site was designated on the lead molecule, a 
single-round optimization by AutoT\&T2 was adequate. Choosing the single-round optimization in this test case was also necessary to be compatible with LUDI.

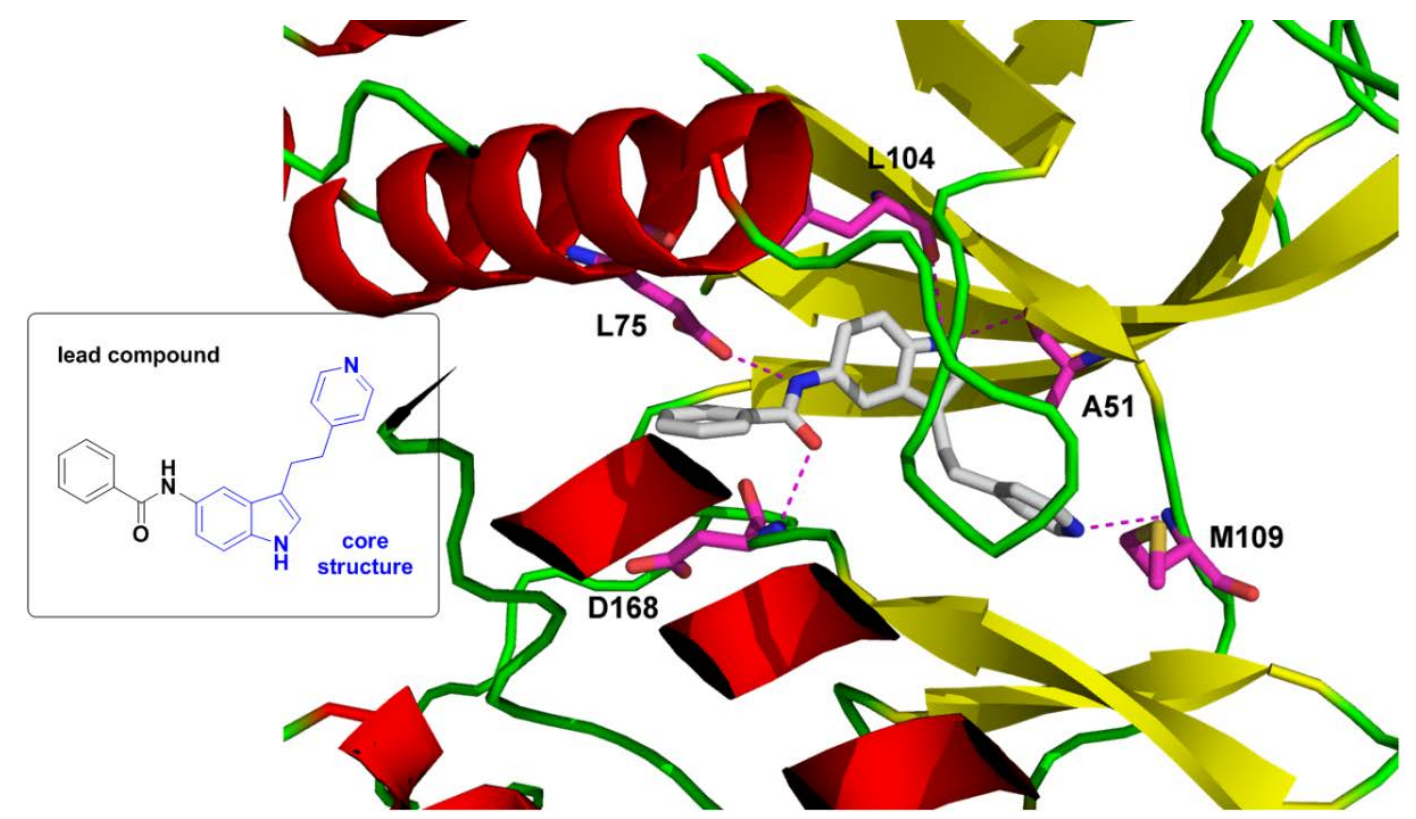

Figure S3. Binding mode of the lead molecule (in stick model) inside the binding pocket of p38 MAP kinase (in ribbon model). The p38 MAPK structure was taken from PDB entry 1W82. As for the lead molecule, the structure in blue was the core fragment to keep; while the benzoic amide moiety was to be optimized.

To set up the job for LUDI, the same site on the lead molecule was designated for growing fragments. The built-in library of 1166 fragments of LUDI was applied to this job. The maximal number of "fit attempts" was set to 10,000. The maximal RMSD value from the optimal protein-ligand interaction was set to 1.0 Å. Protein-ligand interactions were evaluated by the LUDI scoring function, where the weight factors for lipophilic and polar interactions were set to 1.0. Note that the "De Novo Evolution" module in the Discovery Studio software is designed to conduct single-round fragment growing, i.e. installing different fragments onto the designated optimization site.

To set up the job for MUSE, the same site on the lead molecule was designated for growing fragments. The "reaction-driven" genetic algorithm was employed in this job. According to the descriptions in the MUSE user manual, this algorithm considers 87 chemical reactions and about 20,000 reagents to assess the synthetic feasibility of generated ligand molecules. A major difference between MUSE and LUDI in terms of running mode is that MUSE does not allow single-round fragment growing. MUSE replies on a genetic algorithm (GA) procedure to control fragment growing. The minimal number of GA generation allowed by MUSE is 25. Thus, we set the number 
of GA generation to 25 in this test case. The GA population was set to 20 in order to save CPU time since MUSE was kind of slow. The Surflex scoring function was used to evaluate protein-ligand interactions.

The outcomes of this test case are summarized in Table S3 below. AutoT\&T2, LUDI and MUSE generated 45, 26, and 160 new ligand molecules, respectively. These three sets of molecules consist of 28, 9, and 101 molecular frameworks as judged by the molecular framework detection algorithm in AutoT\&T2 (see descriptions in the main text). The CPU time consumed by AutoT\&T2, LUDI, and MUSE to complete the job were 34 seconds, 39 seconds, and about 9 hours, respectively. In this test case, AutoT\&T2 and LUDI performed the same type of job, i.e. single-round fragment growing, and they consumed roughly the same amount of CPU time. But AutoT\&T2 produced structurally more diverse results than LUDI. MUSE produced a greater number of ligand molecules as the result of a 25-round fragment growing instead of a single round. However, MUSE consumed over 1000 folds more CPU time than AutoT\&T2 or LUDI to produce only a few folds more molecular structures.

To assess the "drug-likeness" of the ligand molecules produced by AutoT\&T2, LUDI, and MUSE in this test case, five physiochemical descriptors were computed, including molecular weight, number of hydrogen bond acceptors, number of hydrogen bond donors, number of rotatable single bonds, and octanol-water partition coefficient $(\log P)$. One can see that the ligand molecules generated by LUDI were rather small with an average molecular weight below 300. It indicates that the built-in fragment library used by LUDI mainly consists of small fragments, such as simple functional groups, alkyl groups of fewer than five heavy atoms, simple aromatic rings, and so on. In contrast, the average molecular weight of the ligand molecules generated by AutoT\&T2 is about 56\% higher than those given by LUDI, i.e. 432 vs. 276. The building blocks used by AutoT\&T2 in constructing molecular structures are truncated from organic molecules. They are not limited to simple functional groups or fragments. Therefore, more sophisticated chemical structures can be constructed by AutoT\&T2. All of the ligand molecules generated by LUDI are in accordance with Lipinski’s "rule-of-five” due to their small size. Almost all ligand molecules (only one exception) generated by AutoT\&T2 are also in accordance with Lipinski’s "rule-of-five”, although they are generally more sophisticated than those given by LUDI. The ligand molecules generated by MUSE are generally larger in size with an average molecular weight over 500 . This is because they are the result of multi-round fragment growing. Not surprisingly, a significant fraction (63\%) of the ligand molecules generated by MUSE violates the "rule-of-five". We believe that if fewer round of operation was allowed, the "drug-likeness" of the ligand molecules generated by MUSE could be improved. 
In addition, synthetic feasibility of the ligand molecules generated by AutoT\&T2, LUDI, and MUSE was assessed by applying the "Ligand Properties" module in the MOE software (version 2014.10, Chemical Computing Group Inc.). This module provides a synthetic feasibility score between $0 \%$ to $100 \%$ for a given molecular structure. Here, a score of $100 \%$ denotes the best synthetic feasibility while any score below it indicates some potential problem in this aspect. As shown in Figure S3, all of the ligand molecules generated by LUDI have a synthetic score of $100 \%$ due to their simple chemical structure. Among the 45 ligand molecules generated by AutoT\&T2, only one has a synthetic score below 100\%. This result suggests that the RECAP rules in AutoT\&T2 for controlling synthetic feasibility are helpful. In contrast, 40 of the 160 (25\%) ligand molecules generated by MUSE have a synthetic score below 100\%. It is interesting to observe this result since MUSE in theory has the most sophisticated control on synthetic feasibility during structural construction. This reflects the fact that the molecular structures generated through fragment assembling by a conventional build-up method is prone to problems in synthetic feasibility.

Table S3. Basic information of the generated ligand molecules by AutoT\&T2, LUDI and MUSE in this test case

\begin{tabular}{|c|c|c|c|}
\hline & AutoT\&T2 & LUDI & MUSE \\
\hline $\begin{array}{l}\text { Number of generated } \\
\text { ligand molecules }\end{array}$ & 45 & 26 & 160 \\
\hline $\begin{array}{l}\text { Number of unique } \\
\text { molecular frameworks }\end{array}$ & 28 & 9 & 101 \\
\hline Molecular weight & $432 \pm 46^{a}$ & $276 \pm 24$ & $548 \pm 114$ \\
\hline $\begin{array}{c}\text { Number of H-bond } \\
\text { donors }\end{array}$ & $1.9 \pm 0.7$ & $2.0 \pm 0.5$ & $1.8 \pm 0.8$ \\
\hline $\begin{array}{l}\text { Number of H-bond } \\
\text { acceptors }\end{array}$ & $3.3 \pm 1.3$ & $1.2 \pm 0.4$ & $3.7 \pm 1.5$ \\
\hline $\begin{array}{l}\text { Number of rotatable } \\
\text { bonds }\end{array}$ & $7.4 \pm 1.4$ & $4.8 \pm 1.0$ & $8.7 \pm 3.1$ \\
\hline $\operatorname{logP}$ value ${ }^{b}$ & $4.6 \pm 0.9$ & $2.9 \pm 0.8$ & $6.2 \pm 1.5$ \\
\hline $\begin{array}{l}\text { Number of molecules } \\
\text { good for "rule-of-five" }\end{array}$ & $44(97.8 \%)^{c}$ & 26 (100\%) & 58 (36.3\%) \\
\hline $\begin{array}{c}\text { Synthetic feasible } \\
\text { molecules }\end{array}$ & 44 (97.8\%) & 26 (100\%) & 120 (75\%) \\
\hline
\end{tabular}


Based on the result obtained in this test case, we conclude that running at the same level of efficiency, AutoT\&T2 is able to produce structurally more diverse ligand molecules than LUDI in this single-round fragment growing job. Note that providing structurally diverse designs is the primary aim of de novo design programs because they are normally employed as "idea generator" in structure-based drug design. Quality of the ligand molecules generated by AutoT\&T2, in terms of "drug-likeness" and synthetic feasibility, is as good as those given by LUDI. Moreover, AutoT\&T2 is able to conduct multi-round optimization automatically while it is technically not possible for LUDI to conduct this type of job. As for MUSE, it consumed a much longer running time in this test case because it conducted a multi-round optimization job. As result, it generated more diverse and sophisticated ligand molecules. But the downside is that the quality of the ligand molecules given by MUSE is lower due to their structural complexity. In summary, AutoT\&T2 has proven itself as a useful computational tool for structure-based drug design in this test case. 
Part III. Additional Results Given by AutoT\&T2 in the Three Test Cases Described in the Main Text

Figure S4. Distributions of five molecular properties of the generated ligand molecules by AutoT\&T and AutoT\&T2 through a five-round optimization job (Test case 1, ACE)

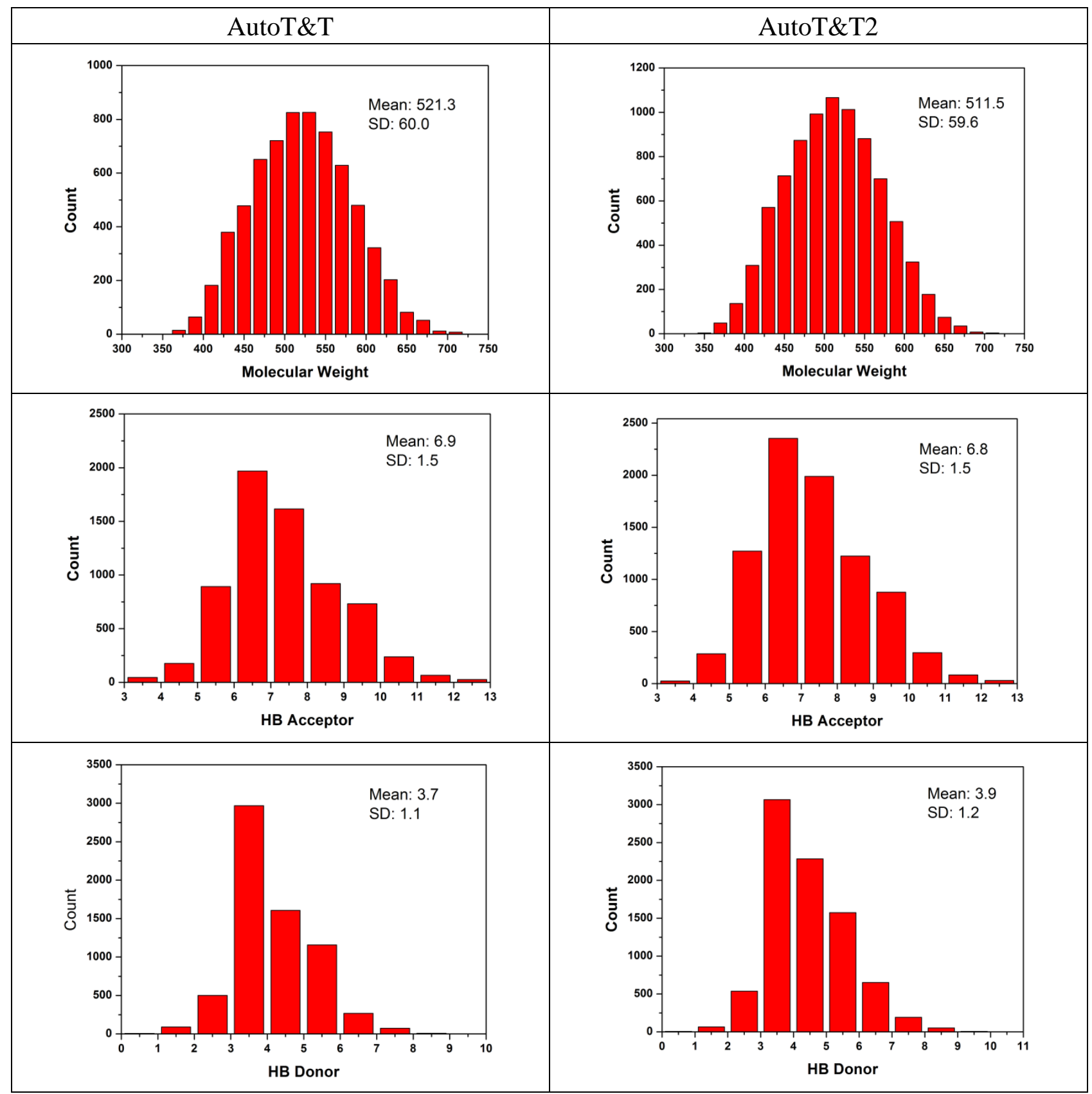




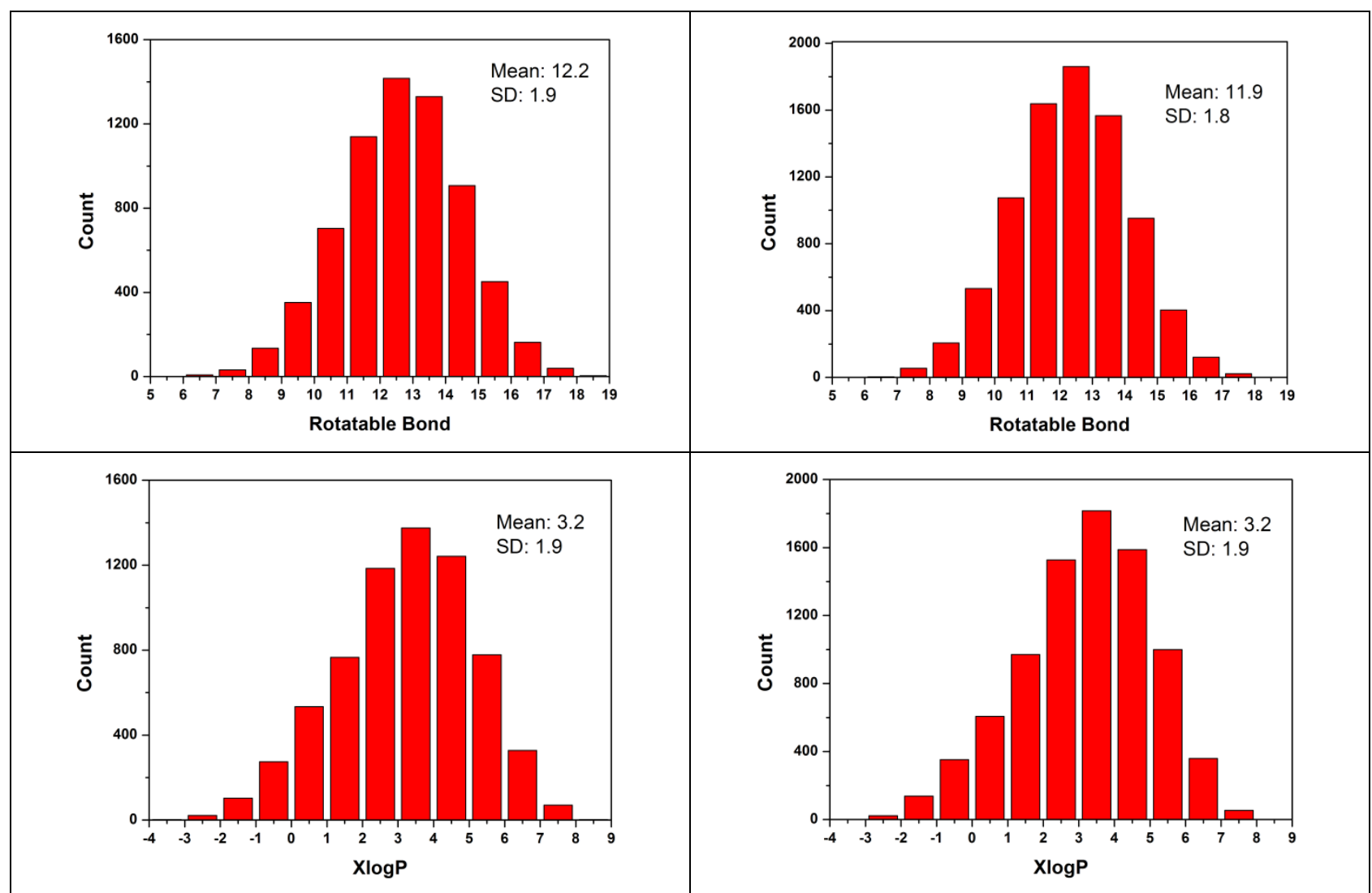


Table S4. VEGFR-2 Inhibitors Retrieved from DrugBank 4 and PDBbind v.2014 (The 12 shaded inhibitors were actually used as inputs in the test case of multi-thread optimization)

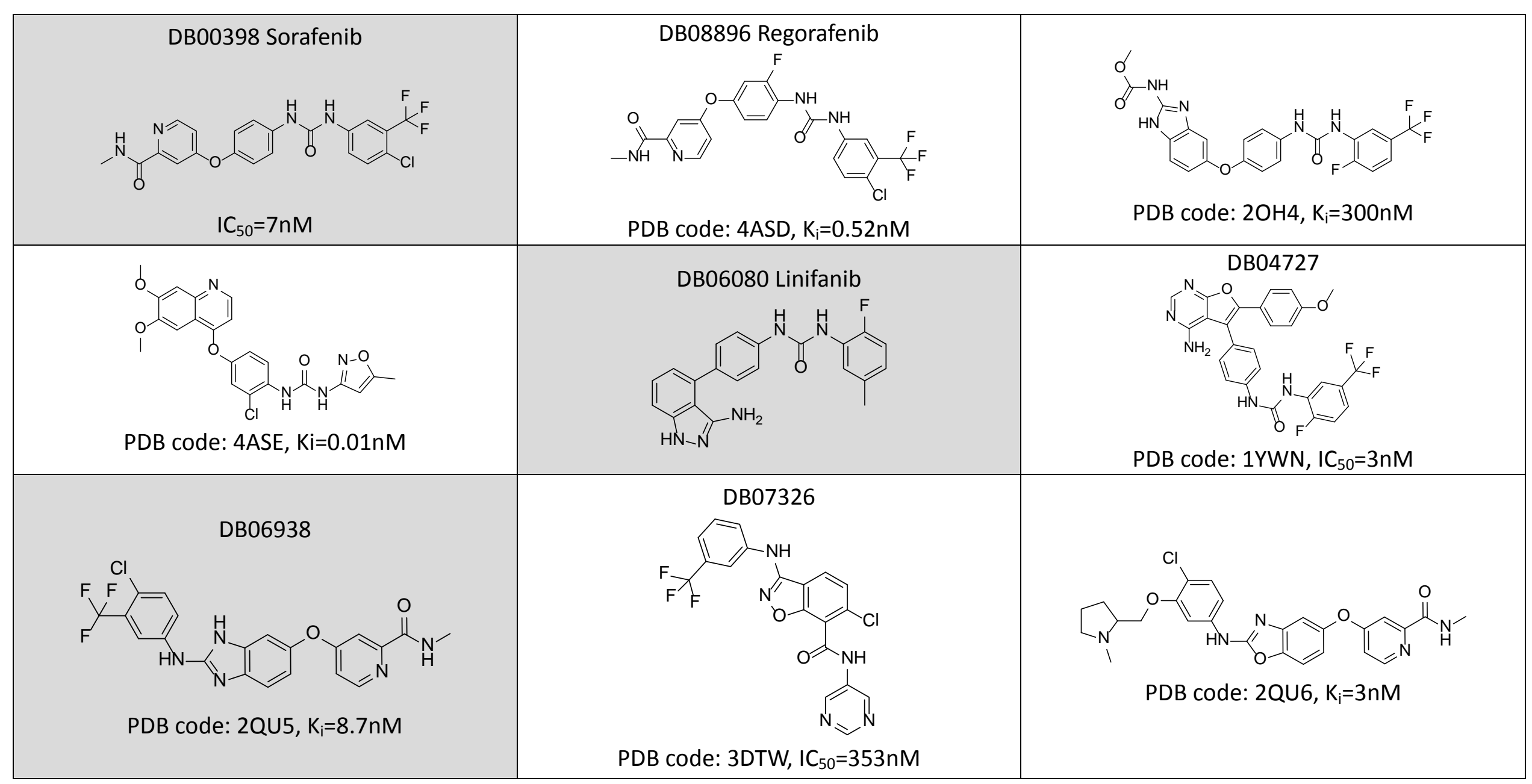




\begin{tabular}{|c|c|c|}
\hline PDB code: $1 \mathrm{Y} 6 \mathrm{~B}, \mathrm{IC} 50=38 \mathrm{nM}$ & $\mathrm{PDB}$ code: $1 \mathrm{Y} 6 \mathrm{~A}, \mathrm{IC}_{50}=22 \mathrm{nM}$ & DB06626 Axitinib \\
\hline PDB code: $3 C P 9, C_{50}=48 n M$ & PDB code: $3 C P C, I C=5 \mu M$ & DB08519 \\
\hline (NB06589 Pazopanib & PDB code: $2 \mathrm{P} 2 \mathrm{H}, \mathrm{IC}_{50}=68 \mathrm{nM}$ & DB08042 \\
\hline
\end{tabular}




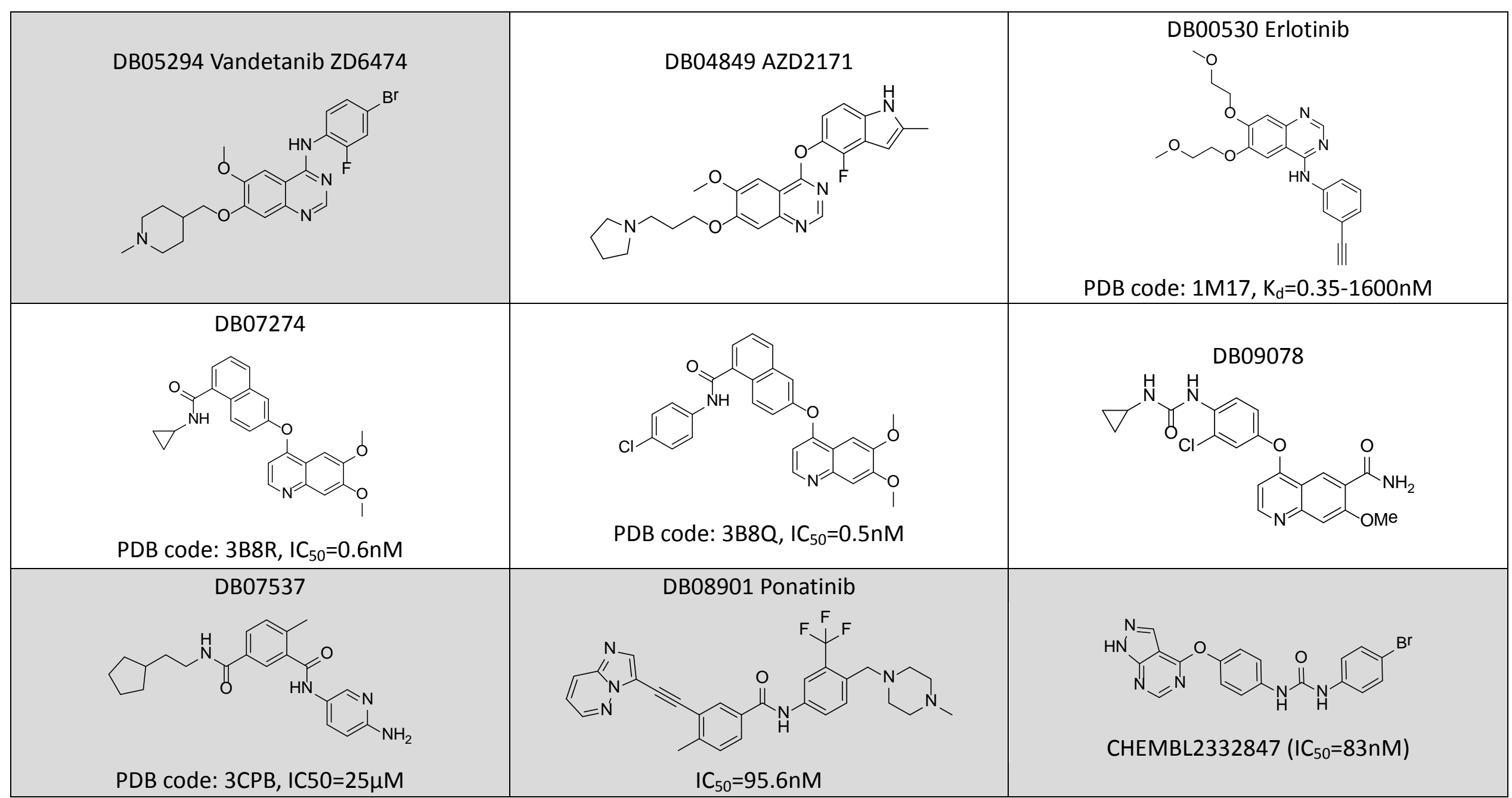




(n)


Table S5. Chemical structures of the ligand molecules generated by AutoT\&T2 in the third test case

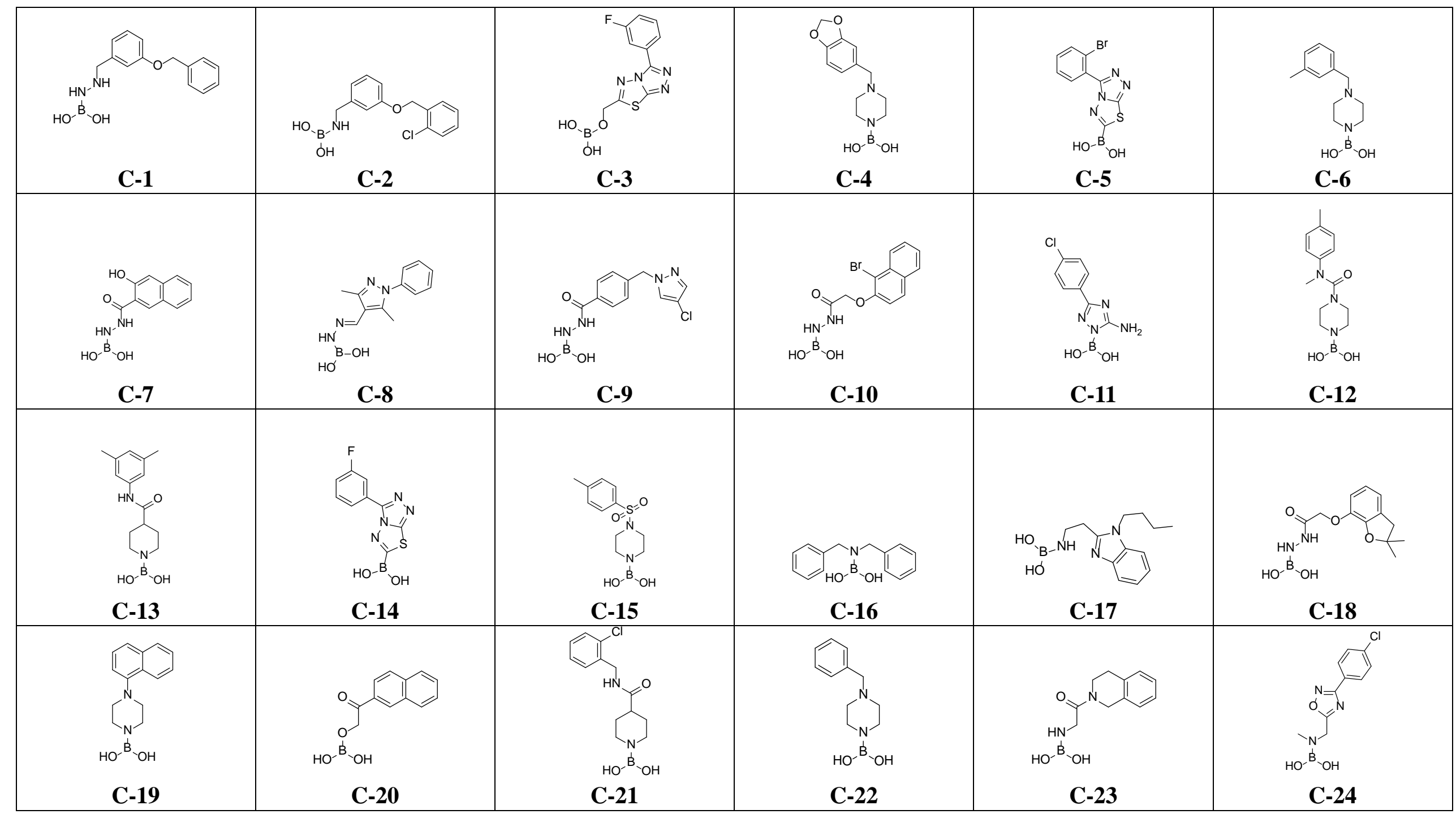




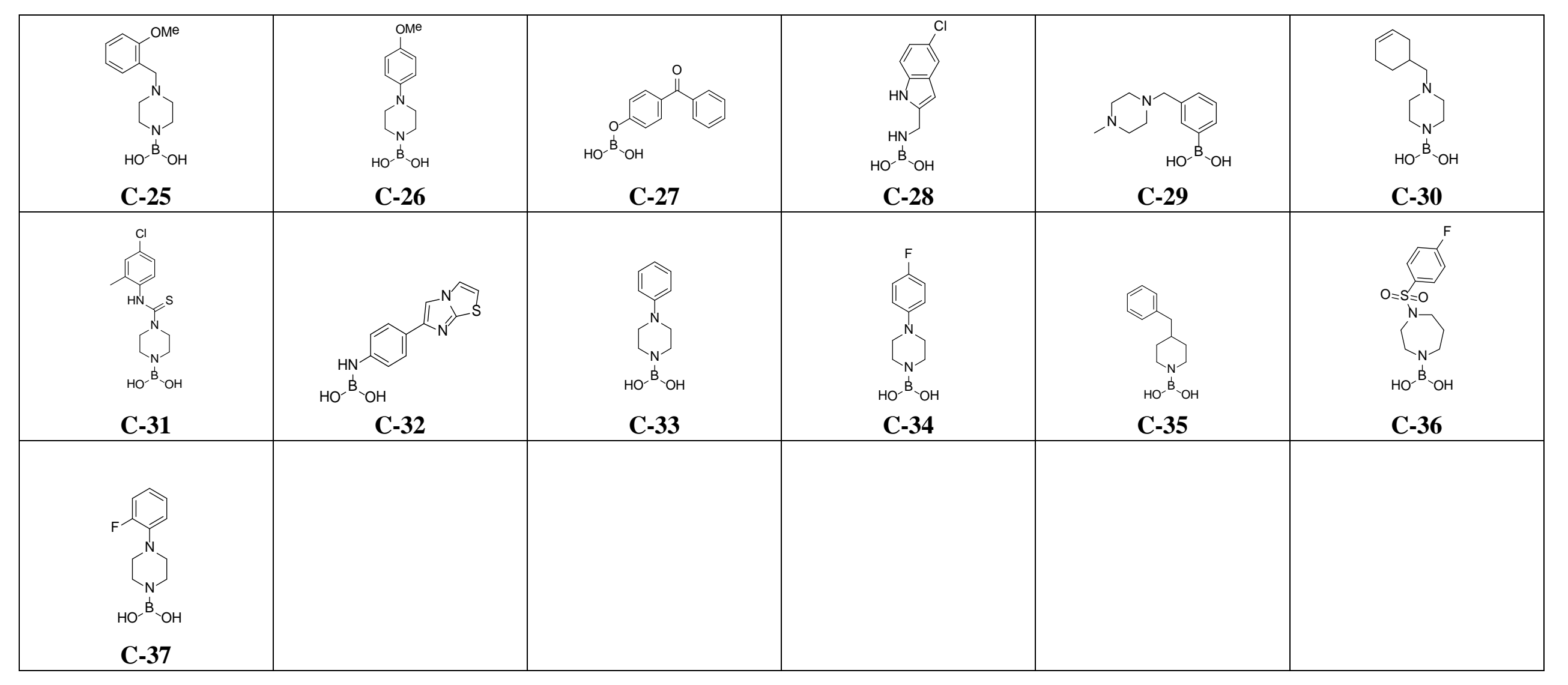




\section{REFERENCES}

(1) Gehlhaar, D. K.; Verkhivker, G. M.; Rejto, P. A.; Sherman, C. J.; Fogel, D. B.; Fogel, L. J.; Freer, S. T. Molecular Recognition of the Inhibitor AG-1343 by HIV-1 Protease: Conformationally Flexible Docking by Evolutionary Programming. Chem. Biol. 1995, 2, 317-324.

(2) Cheng, T.; Li, X.; Li, Y.; Liu, Z.; Wang, R. Comparative Assessment of Scoring Functions on a Diverse Test Set. J. Chem. Inf. Model. 2009, 49, 1079-1093.

(3) Li, Y.; Han, Li.; Liu, Z.; Wang, R. Comparative Assessment of Scoring Functions on an Updated Benchmark: 2. Evaluation Methods and General Results. J. Chem. Inf. Model. 2014, $54,1717-1736$.

(4) Lewell, X. Q.; Judd, D. B.; Watson, S. P.; Hann, M. M. RECAP-Retrosynthetic Combinatorial Analysis Procedure: A Powerful New Technique for Identifying Privileged Molecular Fragments with Useful Applications in Combinatorial Chemistry. J. Chem. Inf. Comput. Sci. 1998, 38, 511-522.

(5) Rogers, D.; Brown, R. D.; Hahn, M. Using Extended-Connectivity Fingerprints with Laplacian-Modified Bayesian Analysis in High-Throughput Screening Follow-Up. J. Biomol. Screen. 2005, 10, 682-686.

(6) Baber, J. C.; Lowe, R.; Saunders, J.; Feher, M. Automated Generation of Turn Mimetics: Proof of Concept Study for the MC4 Receptor. Bioorg. Med. Chem. 2012, 20, 3565-3574.

(7) Liu, Q.; Masek, B.; Smith, K.; Smith, J. A Tagged Fragment Method for Evolutionary Structure-Based de novo Lead Generation and Optimization. J. Med. Chem. 2007, 50, 5392-5402.

(8) Li, Y.; Zhao, Y.; Liu, Z.; Wang, R. Automatic Tailoring and Transplanting: A Practical Method that Makes Virtual Screening More Useful. J. Chem. Inf. Model. 2011, 51, 1474-1491.

(9) Gill, A. L.; Frederickson, M.; Cleasby, A.; Woodhead, S. J.; Carr, M. G.; Woodhead, A. J.; Walker, M. T.; Congreve, M. S.; Devine, L. A.; Tisi, D.; O'Reilly, M.; Seavers, L. C.; Davis, D. J.; Curry, J.; Anthony, R.; Padova, A.; Murray, C. W.; Carr, R. A.; Jhoti, H. Identification of Novel p38-alpha MAP Kinase Inhibitors Using Fragment-Based Lead Generation. J. Med. Chem., 2005, 48, 414-426. 